\title{
Psychiatry in Vietnam: a personal impression
}

\author{
John Wallace
}

After the fall of South Vietnam to North Vietnamese forces in 1975, Vietnam has remained virtually isolated from the rest of the world. With the global political changes of the past 10 years, the Hanoi Government succeeded in reducing Vietnam's international isolation in part by attempting to open the country's door to foreign visitors. The country stretches along the eastern coast of the Indo-Chinese peninsula and is slightly larger than Italy. It is 'S-shaped', broad in the north and south and very narrow in the centre (Fig. 1). The country's main cultivated areas are the Red River delta in the north and the Mekong delta containing Ho Chi Min city (Saigon) in the south.

I would like to describe my brief experience of psychiatry in the Mekong delta region of Vietnam. I was in the Can Tho province of Vietnam in November 1996 and I was privileged to be invited by the Director of the Province's Department of Health to see how the psychiatric service operated in the Mekong delta region.

I flew into Saigon Airport, and travelled to Can Tho, a province situated south of Saigon on the Mekong delta. This province contains some two million inhabitants. Here I saw a rural and, by Western standards, a very impoverished area engaged mainly in farming and fishing. For health and administrative purposes the province is divided into numerous communes, each commune containing approximately 10 villages. Psychiatry is strongly community based in Can Tho, with an emphasis on the psychiatric patient being cared for by his family at home in his native village or commune.

I discovered that there are only five Vietnamese psychiatrists, French and Dutch trained, looking after the psychiatric needs of Can Tho's two million inhabitants. The five consist of a clinical director and his four assistants who have access to $\mathbf{2 5}$ acute in-patient psychiatric beds.

I was told that the psychiatric service in Vietnam began in 1993. Vietnamese psychiatrists believe that "the community must work for the patient". The service is community based with the psychiatric team visiting the patient and their family in his/her village at least every six months. I travelled with the psychiatric team to a

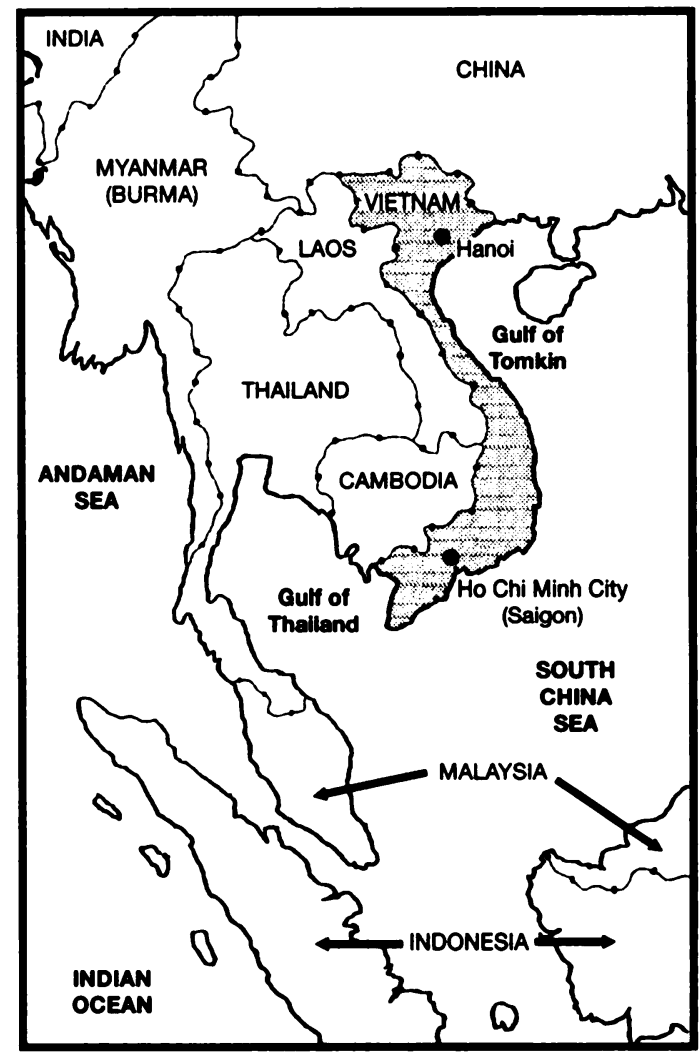

Figure 1. Map of Vietnam.

number of such villages by canoe; the canoe was donated by a European benefactor. At village meetings organised by the team the villagers were educated on how to recognise mental illness rather than simply seeing it in spiritual or religious terms. In the village the family of the psychiatric patient is educated about the nature of the illness and how to deal with it. One family member is paid 4000 Dông (50p) each six months to act as the patient's keyworker. Community care is made possible by the nature of the extended family in Vietnam and also by the 
long and strong tradition of the villagers in looking after a sick family member or soldier.

Patients suffering from schizophrenic illness get priority from the psychiatric service. Depression, I was told, is grossly under-diagnosed as admitting depression involves serious loss of face' within the culture. Psychiatrists that I spoke to regretted the lack of access to the skills of behaviour therapists and clinical psychologists in dealing with the wide range of anxiety disorders that they encounter. The Government does not see alcohol or substance misuse as part of psychiatry's remit at present, but doctors working locally are very concerned about the extent of these problems. The division between neurology and psychiatry is blurred in Vietnam with psychiatrists looking after the needs of neurology patients. Lack of funding for the treatment of epilepsy is a major problem in Can Tho. Because of the nature of the Mekong delta many patients with untreated epilepsy drown each year while working on the river. There is no learning disability service yet south of Saigon but a grant of US\$4000 'start-up money' from a Scandinavian institution has meant that a service for learning disabilities is being developed in seven communes each year, a fraction of the total.

Treatment of the psychiatric patient emphasises social skills training and utilises family meetings. I was told by a psychiatrist that the family is taught to 'treat the patient'. In one remote village I saw the doctor point out the danger of harsh criticism and over involvement with a patient with schizophrenia. He also discussed the value of medication and the drawbacks of physical restraint. Unfortunately, in another village I saw one young patient with schizophrenia kept in chains by his family just prior to his been taken on to a treatment programme by the psychiatric team.

Medication must often be bought by the family at the local market. In a country where a psychiatrist earns the equivalent of US\$50 a month it is hard for a rural family of a patient to obtain medication. Can Tho province has no electronconvulsive therapy machine, little access to laboratory tests and most importantly, very few books outside the main city. I was persistently asked by doctors for old psychiatry textbooks. One doctor in Can Tho city receives the British Journal of Psychiatry and meetings are held to discuss its contents.

Can Tho province is just one of the 13 provinces of the Mekong delta region. This large area contains some thirty million people and lies on a peninsula jutting into the South China Sea bounded by Cambodia to the north east and Saigon directly north. I had the opportunity to travel to some of the more remote provinces. I was told that these provinces are currently attempting to replicate the community care model being developed in Can Tho province. Staff view the fact that the services are being developed from scratch as a positive and unique opportunity. Unfortunately, I noted that the further an area is from Saigon city, the fewer facilities, books and medication that are available for service development. Travel was difficult and facilities sparse in these more remote regions. I was struck, however, by the enthusiasm and commitment of staff running the newly developed psychiatric service. In one rural psychiatric in-patient unit I noted that the patients wore white uniforms and the staff dressed casually. The atmosphere was always extremely cordial and relaxed with the staff very knowledgable about psychiatry.

Despite its short history and lack of funds, psychiatry in Vietnam has managed to introduce a psychiatric service that draws on the more recent social and psychological trends towards mental illness and apply them in a community setting. Even with the lack of funds the service has been systematically extended to different patient groups by enthusiastic and knowledgeable personnel. Utilising the advantages offered by a supportive extended family network a community-based psychiatric service is being developed which is now beginning to have a significant impact on psychiatric disorder in this damaged but very energetic country. The success of the service within Vietnam has positive implications for the region as a whole.

\section{Acknowledgements}

Special thanks to Dr Nguyen Mong Giao, Director of the Neurologic Psychiatric Centre, 71 Ly Tu Trong Street, Can Tho City, Vietnam and Dr Vincent Kenny, Department of Health and Tropical Medicine, Royal College of Surgeons in Ireland, Dublin 2.

John Wallace, Clinical Psychologist and Registrar in Psychiatry, Mid-Western Health Board. St Camillus' Hospital, Shelbourne Road, Limerick 